\title{
Ricordo e oblio nelle donne della Commedia
}

Mémoire et oubli chez les femmes de la Comédie

Memory and Oblivion in the Women of Comedy

\section{Valter Leonardo Puccetti}

\section{OpenEdition}

\section{Journals}

Edizione digitale

URL: https://journals.openedition.org/cei/9900

DOI: 10.4000/cei.9900

ISSN: 2260-779X

\section{Editore}

UGA Éditions/Université Grenoble Alpes

\section{Edizione cartacea}

ISBN: 978-2-37747-304-5

ISSN: $1770-9571$

\section{Notizia bibliografica digitale}

Valter Leonardo Puccetti, «Ricordo e oblio nelle donne della Commedia», Cahiers d'études italiennes

[Online], 33 | 2021, online dal 01 octobre 2021, consultato il 13 octobre 2021. URL: http:// journals.openedition.org/cei/9900 ; DOl: https://doi.org/10.4000/cei.9900

Questo documento è stato generato automaticamente il 13 octobre 2021.

(c) ELLUG 


\title{
Ricordo e oblio nelle donne della Commedia
}

\author{
Mémoire et oubli chez les femmes de la Comédie \\ Memory and Oblivion in the Women of Comedy
}

Valter Leonardo Puccetti

A Bennie e al suo giro al revés.

1 Non risulta facile smontare 'ideologicamente' le letture romantiche o, per il meno, desanctisiane dei personaggi femminili della Commedia. Battuta in breccia ormai da tempo la deteologizzazione ovvero la depenalizzazione delle esaltanti scorie di umanità della donna infernale, purgatoriale o finanche paradisiaca quando essa guarda dietro di sé (l'eternità, hegelianamente ${ }^{1}$, come piedistallo delle passioni terrestri e direi quasi della muscolatura spirituale del personaggio), resta tuttavia un Kernpunkt, un punto chiave di resistenza che caratterizza il femminile nel poema dantesco e che lo proietta sullo schermo, che parrebbe anacronisticamente romantik, del non finito e della Sehnsucht.

2 Rimuovere il riscontro oggettivo o alterare, nella lettura del poema dantesco, le isotopie dell'insolvenza, del rimpianto o della deprecazione dell'inconcluso per le figure femminili, non sarebbe interpretativamente onesto e soprattutto impoverirebbe catastroficamente lo spessore e l'irraggiamento del testo. Non si tratta di un effetto impressionistico di lettura o di un difetto orientato del filtro di lettura, quale ad esempio Gianfranco Contini rimproverò a Attilio Momigliano («l'impression sensible et la mezza tinta (jamais l'idée!) »'), commentatore dantesco il più intrigato dalle atmosfere malinconiche, dalla nostomania del non-finito: è che le donne della Commedia alludono in effetti costantemente a un'incompiutezza, a una distanza non colmata o a un tradimento del compito di genere (il che riconduce comunque a una base di struggente inadempienza).

3 Credo pertanto che obiettivo della critica dantesca sia non esorcizzare, per tema di cedimento sentimentale e di scarso rigore storico, il dato suddetto, quale esemplificheremo nell'analisi, ma inquadrare esso nella visione organica della realtà e 
delle sue istanze da parte di Dante: insomma, di strutturare il dato. E la misura di questa irresoluzione (nel senso di non soluzione, nel senso di riserva di un margine) del personaggio femminile medio della Commedia la fornisce il tempo di vita a fronte di quello eterno, sia per insufficienza di fruizione sia per irrevocabilità di scelta. Ciò potrebbe sembrare un truismo e mancare di specificità: non è per caso la condizione del cristiano alla prova, del cristiano al punto, quella che fulmina peccatori e penitenti, entro le terzine dantesche, in gesti fatali, in pose eloquenti di destino, si potrebbe obiettare?

La risposta, dopo tutto, è no: c'è una singolarità d'azione e di responsabilità del maschile che, nel cambio di genere, sfuma invece in connotazione collettiva, in una fragilità essenziale e in una perdita d'ufficio che appunto viene poi a minare l'intera collettività. Ex una disce omnes, si avrebbe voglia di parafrasare: se si preferisce, mozartianamente, così fan tutte. Ma l'accento batte sulla risposta di genere, sul 'tutte'. Perciò, in tanti destini femminili dentro il poema, la dialettica temporale si converte in epocalità, in ciclo del dissesto etico della polis ${ }^{3}$, della cui stabilità Dante rendeva garante e metronoma proprio la donna, la donna-famiglia, la donna-amalgama, la cui individualità (in termini postdanteschi: la cui psicologia) è non solo inconferente ma se insorgente allora peccaminosa. Maria, la Santa Vergine, rappresenterà nel Paradiso, dapprima con gli exempla ammonitori, per «visibile parlare» ${ }^{4}$, e poi con la sua muta fissità, viva tuttavia di una tensione intercedente (qualcuno ha evocato ecfrasi figurativa da arte di mezzo, in fuoriuscita dal bizantinismo, tra Duccio e Cavallini) ${ }^{5}$ in Empireo, il modello della femminilità della rinuncia, del sacrificio, dell'abdicazione volontaria alla singolarità.

5 Ma siccome l'ontogenesi nella Commedia riflette narrativamente la filogenesi, con punte di drammaticità catartica che trovano apice nel paradiso terrestre, la dinamica dell'insolvenza coinvolge la stessa vicenda del viator: Beatrice e Matelda svelano l'insolvenza del poeta-personaggio, la sua distanza dal dover essere, che il viaggio oltremondano deve colmare proprio come restituzione della memoria e nel dolore che a quel processo di recupero è connesso ${ }^{6}$. In quel caso il risucchio del tempo, a ritroso verso il bivio di sviamento, è occasionato da una figura di donna giudicante: è Beatrice, riflesso mariano, che torna dal passato e metra l'inadempienza, conta i passi mancanti. Dante, per dirla con sue parole altrove, repetendo le volte tristo impara. Il fallito incontro col femminile virtuoso ovvero il misconoscimento di esso, in un periodo della vita di Dante, diventano la riprova di un valore di genere che sarà uno degli acquisti fondamentali del viaggio nell'oltremondo, fino alla cruciale risoluzione dello sguardo negli occhi di Maria.

6 Il nostro, di viaggio, dentro il poema dantesco, sui luoghi testuali della femminilità, può invece cominciare ovunque e terminare in ogni dove, a illustrare gli assunti, che poi sono desunti, sopra esposti. Sarebbe facile, e dunque cediamo pure alla facilità, partire dai canti paralleli - riconosciuti come tali ben prima che Barański 'scoprisse' le simmetrie di sezione fra le cantiche ${ }^{7}$ - che implicano tre donne infedeli alla quinta stazione di ogni cantica: Francesca, Pia, Piccarda. Nel caso di Piccarda l'apparizione dell'anima ha luogo in effetti al terzo canto ma propaggina fino al quinto, sì che la corrispondenza è recuperata sulla durata. Il cinque è il numero dei sensi e quindi dell'esistenza sensibile in simbologia cristiana, ma a partire da Ildegarda di Bingen ${ }^{8}$ rinnova, in ambito cristiano, la simbologia pitagorica nuziale (il cinque risulta dalla somma del primo numero pari e del primo dispari dopo l'uno perfettissimo). A seconda 
della direzione della punta del pentagramma (il numero è del resto in equilibrio all'interno della decina) l'uomo inclina verso l'alto o verso il basso, verso il bene o verso il male: del resto la prima lettera ai corinzi $(\mathrm{XIV}, 16)$ induce a preferire cinque parole con intelligenza che diecimila col dono delle lingue e il laconismo di Pia e di Piccarda sul tragico clou delle loro esistenze sembra raccogliere il monito paolino, Francesca, nella sua logorrea giustificazionista, disattenderlo. Cinque sono poi le pietre che Davide raccoglie nel torrente e ripone nel secchio del latte e con una (l'unità che porta a compimento la Legge) di esse abbatterà Golia: Agostino ci dice pertanto che Davide le ha cavate sotto la corrente dei sensi, in mezzo al flusso dell'effimero ${ }^{10}$. L'uomo scava la salvezza nell'osservanza della Legge (cinque sono i libri di Mosè) sotto l'incostanza, sotto la mutevolezza illusoria della vita sensibile.

7 Le tre donne, in scala ascendente (la trinità dei canti riservata nel Paradiso al 'caso Piccarda', terzo quarto quinto, riscatta definitivamente l'ambiguità ancipite della pentuplicità) ${ }^{11}$, verificano la costanza e l'adeguatezza della loro partecipazione fusionale, della loro capacità di catalisi nuziale. Tutte e tre lasciano residuo: tragicamente nel caso di Francesca, con velatura della beatitudine nella stessa Piccarda, sospesa Pia, nell'antipurgatorio (se è lì, solo il pentimento in articulo mortis l'ha salvata dal gravame di peccato mortale e i commentatori antichi che spettegolano sulla sua verve adulterina sono con Dante molto probabilmente in sintonia migliore di noi), in bilico fra lo ieri fuggevole eppure ancora doloroso e il domani che s'insemprerà. Tutte e tre sono illuminate da un ricordo che presto si fa ombra, tenebra soverchiante di uomini che fanno violenza («chi a vita ci spense», Inf. V, 107; «colui che 'nnanellata pria», Purg. V, 135; «Uomini poi, più a mal ch'a bene usi», Par. III, 106): nel caso di Francesca e di Piccarda «tempo felice» (Inf. V, 122) e «dolce chiostra» (Par. III, 107) appaiono dietro stimolo d'indagine del viator (con ugual movenza: «Ma dimmi: al tempo d'i dolci sospiri», Inf. V, 118; «Ma dimmi: voi che siete qui felici», Par. III, 64), mentre Pia si fa avanti da sola portando la luce della «gemma» (Purg. V, 136) con cui fu "'nnanellata», unico episodio riemerso notabile tra il farsi e il disfarsi della sua vita, come lei incide nella sua epigrafe in terzine ${ }^{12}$.

8 Non si tratta di tre madeleines proustiane: su ciò la critica 'insensibile' ha ben diritto di impuntarsi. Il ricordo castiga, forma una cicatrice, dichiara una sconfitta che a seconda delle tre donne è più o meno saldata. Lo sguardo indietro non solo definisce un naturale intervallo temporale fra la dimensione dell'eterno (definitiva per Francesca e per Piccarda, promessa per Pia) e quella del tempo caduco e sigillato nel passato: individua anche una beanza in quel passato, un'inconcludenza, che impedì fedeltà. Per Francesca è l'intervallo fra la durata senza tempo in cui siede la marina «dove il Po discende / per aver pace co' seguaci sui» (Inf. V, 98-99) e la compressione insostenibile del tempo («quel giorno più non vi leggemmo avante», 138) del «punto» che la «vinse» (132). Per Pia, il «pria» (135) di un giorno che riassume una vita (il gerundio «disposando», inframezzato tra participio e ausiliare posposto, produce retoricamente una coacervatio, una rutilanza, un eccesso di superficie, che contrasta con lo squallore desolato della chiastica cornice di «Siena mi fè, disfecemi Maremma» [134]) apre una frattura indeterminata e fagocitante con un 'poi' che resta off. Piccarda, infine, sublima ma non raccorda la «dolce chiostra» (Par. III, 107) del matrimonio mistico con la reticenza del «poi» di sofferenza («Iddio si sa qual poi mia vita fusi», 108): un clivaggio, perché Piccarda non seppe scegliere il martirio ma solo viverne e depurarne le conseguenze. 
Un distacco, un abbandono segnano la memoria femminile, nella Commedia: mai dolci i ricordi, se ricordi, per le donne in aldilà. La gioia feroce della «sanese» Sapìa, alla disfatta della sua gente a Colle Val d'Elsa, non fa eccezione: è in Purgatorio puntuale ricordo di un momento emblematico di quel peccato, l'invidia, che aveva rischiato di perder Sapìa sulla terra, è quel che lei deve emendare sulla seconda balza del monte di espiazione. Anche in questo caso il peccato femminile viene da rottura di vincolo, da un atto di separazione: Sapìa ha festeggiato il male inflitto alla sua comunità. Il peccato dantesco per eccellenza, di disgregazione del tessuto della polis (in Sapìa puramente ottativo, ma basta: «fui de li altrui danni / più lieta assai che di ventura mia», Purg. X, 110-111), riflette la perdita di orientamento del divino: una bestemmia superba ${ }^{13} \mathrm{ma} \mathrm{di}$ vacua tracotanza, alla Capanèo ("tanto ch'io volsi in su l'ardita faccia, / gridando a Dio: "Omai più non ti temo"»,/ come fé "l merlo per poca bonaccia», 121-123), è il coronamento, in terra, all'augurio disfattista di Sapìa e, in fondo, il «questi che mai da me non fia diviso» (Inf. V, 135), di Francesca che addita Paolo a Dante, poteva parimenti esser letto come blasfemo, come un dispettoso arrogarsi a scelta quel che è invece eterna sentenza di condanna e che nel caso del marito Gianciotto, con superbia abusiva, parimenti Francesca parrebbe rivendicare, a estrema conclusione del suo celeberrimo racconto («Caina attende chi a vita ci spense», 107).

Nell'episodio di Sapìa la profondità del tempo - che nella Commedia è il baratro, per le donne, che hanno scavato tra esse stesse e la loro appartenenza di gruppo sociale, fin dalla cellula familiare fondatrice - è in tanta parte risarcita con altro tempo terreno, quello di chi ha pregato per la nobildonna senese accorciandone l'espiazione, Pier Pettinaio «con sue sante orazioni» (Purg. XIII, 128). Questo, del tempo di memoria o di oblio verso il defunto, è tema portante, sebbene quasi sommerso, nella seconda cantica e forse rimuove o disloca il tema personale per l'autore, che per via del suo esilio è dall'oblio minacciato (come con disarmante naturalezza ha scritto Harald Weinrich) ${ }^{14} \mathrm{e}$ scrive un'opera che in certo modo ne è il sublime antidoto. E concerne soprattutto le mogli o le figlie sopravvissute alle anime incontrate di volta in volta da Dante: Sapìa è l'unico personaggio femminile per il quale si preghi o si sia pregato in terra ed è comunque un frate terziario, un uomo di religione a pregare per lei. Soltanto le donne appaiono cimentate, nel Purgatorio, alla prova della connessione nel ricordo, alla prova della durata o dell'entropia degli affetti. Di fatto, l'armonizzazione sociale cui, per Dante, sono chiamate le donne (il primo dei tre canti di Cacciaguida solennizzerà il concetto col modello di «Fiorenza dentro da la cerchia antica» [Par. XV, 97], come vedremo) è metafora del rapporto con l'eterno: non saper tenere il filo di pensiero col caro trapassato è non altro che secessione dalla salvezza, è promessa di esilio dall'eterno. La lavagna si spartisce così in due colonne, quella delle brave e quella delle cattive: di quelle che mantengono il tempo della fusione, donne che sono come un pleroma, mediazione fra effimero ed eterno in cui sciolgono la loro personalità; e di quelle la cui memoria, montalianamente, altro tempo frastorna, il cui filo si addipana, la cui bussola va impazzita all'avventura...

11 Da un lato Nella, la moglie di Forese che Dante onora in Purgatorio forse (come, dopo Contini ${ }^{15}$, è uso ripetere) in riparazione del salace coinvolgimento nella tenzone giovanile con l'amico: l'insoddisfazione sessuale, nella battutaccia di Dante nel sonetto di aggressione della serie (Chi udisse tossir la malfatata ${ }^{16}$ ), lascia il posto a pii «martiri» (Purg. XXIII, 86), che al lettore del poema possono far pensare, in intertestualità situazionale, alle sofferenze, coperte da reticenza, della cognata di Nella, Piccarda mal 
rimaritata dopo che il suo matrimonio sacro con Cristo era stato rotto. Il pianto della madre di Nella nel sonetto antico («Piange la madre, c'ha più d'una doglia, / dicendo: "Lassa, che per fichi secchi / messa l'avre' in casa il conte Guido!"») si muta, entro il poema, nel «pianger dirotto» (87) della figlia in lutto, la «tosse» da raffreddamento di Nella, per via di «copertoio c'ha cortonese» (Nella veniva poco riscaldata a letto, a dir del giovane Dante...), in «sospiri» (88), che frammezzano i «prieghi devoti» (88) coi quali Nella va accorciando il tempo di permanenza del marito fra gli espianti.

12 La fedeltà, che unisce una polis e di cui la figura della donna è agli occhi di Dante la sacerdotessa laica, ha nemica la sessualità definalizzata, che si consuma nell'imperiosa, isolante istantaneità del piacere ed è perciò disgregatrice dei rapporti: conseguente, sùbito dopo il cammeo riservato a Nella, nel XXIII canto purgatoriale, l'attacco virulento, per bocca di Forese, alle «sfacciate donne fiorentine» (101), che sarà ripreso nel canto succitato di Cacciaguida, il quindicesimo paradisiaco, con fondamentale espansione ideologica alla logica divisoria dei «subiti guadagni» (per dirla col Dante di Inf. XVI, 73) e all'equazione premarxiana fra merci e eros. La lupa dell'avidità del primo canto infernale, che è la mater vitiorum nella costruzione etica del mondo dantesco, permette facile scambio con un simbolo di insaziabilità sessuale: l'ansia irresponsabile e automatica di possesso si riflette (e trae riflesso) nel determinismo famelico del piacere fisico. Nella potenziale disponibilità, secondo Dante, alla mercificazione da parte della donna si realizza l'allegoria vivente del danno sociale, che nella visione dantesca prende sempre forma di desolidarizzazione autistica e irrazionalmente cumulativa. L'anti-Nella, in tale senso, della seconda cantica è la moglie di Nino Visconti, presentato come amico personale dall'auctor al pari di Forese: Beatrice d'Este, che Nino evoca non come moglie o vedova sua ma, emblematicamente, come madre di sua figlia (Giovanna, per la quale soltanto egli affida a Dante le sue preghiere di essere ricordato), e che viene caratterizzata con una terzina, di impianto misogino tradizionale (medieval-monastico voglio dire $)^{17}$, in cui al centro campeggia il vulnus dell'emorragia temporale, per Dante sempre insidia alla e della femminilità: «Per lei assai di lieve si comprende / quanto in femmina foco d'amor dura, / se l'occhio o 'l tatto spesso non l'accende» (Purg. IX, 76-78).

13 La falsa durata della fedeltà femminile (se l'occhio e il tatto spesso l'accende, cioè...) si alimenta, in effetti, della stessa sensualità che avversa apertamente la durata autentica, che è quella dell'eterno, come l'esempio di Beatrice d'Este dovrebbe provare. Beatrice è presto convolata in seconde nozze, con un altro Visconti, stavolta milanese e non pisano e le desolate parole di Nino sembrano alludere a calcolo di potere e di avere: «Non le farà sì bella sepultura / la vipera che Melanesi accampa, / com'avria fatto il gallo di Gallura» (Purg. VIII, 79-81: si profetizza contestualmente post factum la disgrazia e l'esilio del nuovo marito Galeazzo, ma Dante non poteva sapere che nel 1328 Beatrice sarebbe trionfalmente rientrata in Milano col figlio Azzo, il quale rinnovava in Milano la signoria viscontea).

14 Al modello onesto di Nella o a quello deteriore della Estense si possono ricondurre le altre donne lasciate sulla terra dalle anime d'oltremondo della Commedia: Costanza figlia di Manfredi, buona e ligia alla memoria del padre e perseguitata ma vicina a riscatto; Giovanna invece moglie dimentica di Bonconte (e la figlia Manentessa, da Dante conosciuta, non doveva esser più sollecita della madre nella memoria, rientrando nel collettivo generico del verso "Giovanna o altri di me non ha cura») ${ }^{18}$; Alagia Fieschi, che «non trasmutò le bianche bende (Purg. VIII, 74) vedovili come aveva fatto la nipote 
Beatrice d'Este» ${ }^{19}$, moglie e poi vedova di Moroello Malaspina (ammirato «vapor di val di Magra» in Inf. XXIV, 145), che Dante potè conoscere durante la tappa lunigiana del suo esilio e che era esempio intemerato e tuttavia insidiato dalle male donne della famiglia di Adriano V, il quale ad Alagia sola affida il suo buon ricordo («Nepote ho io di là c'ha nome Alagia, / buona da sé, pur che la nostra casa / non faccia lei per essempro malvagia; / e questa sola di là m'è rimasa», Purg. XVI, 142-145); la figlia di Carlo Martello, Clemenza, come la Costanza di Manfredi destinata a reintegro indiretto dei diritti lesi al o perduti dal genitore con la morte.

La continuità del tempo di Provvidenza, che la femminilità virtuosa assicura, la catena di salvezza dalla vita alla morte, esonda invece dal rapporto affettivo e domestico con l'anima intervistata dal viator nel canto ventiquattresimo del Purgatorio e nel caso di Bonagiunta Orbicciani, e tocca il viator medesimo, di cui nessun parente o affetto attuale nel momento del viaggio è fatto cenno dentro il poema. Bonagiunta promette infatti a Dante un soccorso pietoso di donna, tale Gentucca (Purg. XXIV, 37), una sorta di biblica Raab in città ostile (per Dante esule, la Lucca infida ospite guelfa nera) ${ }^{20}$, il filo rosso nell'esilio, o se vogliamo, fuor di metafora biblica, il collante di umanità e di dialogo che per Dante è la donna nella civitas. Figura antigonica di giustizia, anche mi è capitato di scrivere una volta di lei ${ }^{21}$, antitesi della «femmina» (42; notare la vox media, che applicata alla lucchese marca il mero genere, mentre nella razo in prosa della canzone vitanuoviana Donne ch'avete intelletto d'amore, subito dopo evocata da Bonagiunta al v. 51 dell'episodio purgatoriale quale opera di svolta epistemologica per tutta una generazione, è spregiativo ${ }^{22}$, come quasi regolarmente nella Commedia) quale propellente della divisione sociale. Per il viator è come se la sopravvissuta, pietosa sulla terra, venisse dal futuro (è nata da non molto, quando il viaggio dantesco è immaginato, «non porta ancor benda», dice Bonagiunta [43]), una sopravvissuta a lui morto fra i morti: un sogno di rinascita proiettato dai miti giovanili, la staffetta di Matelda.

Altrove, in un saggio sulla presenza di Stazio nella Commedia ${ }^{23}$, mi sono chiesto peraltro se quel poeta antico autorevolmente canonico nel Medioevo, proprio perché insignorito nella cantica purgatoriale e, oltre tutto, con funzione recitante per il lungo excursus sulla procreazione (nel venticinquesimo canto) che assegna teleologia sacra all'atto sessuale, non fosse patrono della funzione ideologica della donna univira e domiseda esemplare nel poema: Stazio, che nella Tebaide ha riservato uno spazio inaudito e irripetuto, nell'epos antico, alla figura femminile, Stazio collezionista di heroides della verecondia e che, esemplificando instancabilmente la formula virgiliana casta pudicitiam servat domus, ha fornito all'etica della conservazione dantesca un mondo da sviluppare.

Il personaggio di Gaia, la caminese figlia del gran Gherardo, fa da cerniera («Per altro sopranome io nol conosco, / s'i nol togliessi da sua figlia Gaia», Purg. XVI, 139-140) tra le due schiere di sopravvissute, le oranti e bene operanti e le dimentiche e tralignanti: il padre di Gaia è ancora vivo quando il viaggio oltremondano di Dante s'immagina avvenuto (non quando Dante scrive) ma in fondo è uomo del passato, è un trapassato ad honorem si vorrebbe quasi dire, che Dante dice unico "saggio» (con Currado da Palazzo e Guido da Castello) «rimaso di quella gente spenta» (132-133), la gente cioè del «paese ch'Adice e Po riga» (115) prima che il mondo divenisse «reo» per «mala condotta» (104-105). Gherardo appartiene infatti a un tempo che fu, quello in cui «solea valore e cortesia trovarsi» (116), un tempo che a Dante appare tanto antico quanto quello di Cacciaguida e che si contrappone a un presente, il «secol selvaggio», di 
devianza e di discordia. Gaia è adesso la vedette della famiglia e secondo Marco Lombardo quasi oscura per fama il padre visto che Marco deve citarla affinché Dante comprenda a qual casata appartenga il Gherardo, prima citato solo per nome. Sul tortuoso riferimento a Gaia i commentatori antichi si erano divisi: tanta era la grazia e persino l'arte poetica di lei (gaia nell'accezione tecnico-provenzale, dunque antonomastica: gioia vitale, misurato saper del sentire e dell'amare), oppure tanto chiacchierati erano i suoi costumi? Come osservano Bosco-Reggio ${ }^{24}$, tutto il discorso di Marco Lombardo, imperniato sul contrasto fra passato e presente, indirizzerebbe verso un'interpretazione disforica: Gaia sarebbe per l'oggi quel che Cianghella, nelle parole di Cacciaguida, è a fronte della «buona Gualdrada» (Inf. XVI, 37: così Jacopo Rusticucci in inferno, Cacciaguida la evoca come «donna» di «Bellincion Berti» [Par. XV, 112-114]) nella Firenze antica, «sobria e pudica» (Par. XV, 99).

Alagia e Nella (sullo sfondo poche altre consimili, che abbiamo citato) sono rare, di marcata rarità, nel tempo presente, che è quasi aggrappato alle preghiere di quelle che hanno conservato le virtù che conservano: non è un bisticcio verbale il mio, denota l'attitudine che Dante attribuisce a queste donne, una resistenza morale, una chiusura solitaria, dolcemente indomita, in un tempio ormai disertato e che esse vigilano tuttavia. Le loro orazioni sono novissima verba: le ultime parole, che sono di ricongiunzione al giudizio ormai imminente (pochi scranni rimangono vacanti in Empireo...). Le 'altre' determinano, invece, la decadenza di un'epoca tutta col loro distacco da un codice nel quale il discorso di Marco Lombardo mostra di far convergere culto della legalità e cortesia, forse in modo sorprendente per chi non abbia capito che Francesca da Rimini delle tavole d'amore cortese fornisce lezione alterata, ingannata e ingannatrice quale è. Il tempo del distacco e dell'abbandono, da parte delle donne, ha prodotto il declino dei tempi, la perdita del tempo antico, di un tempo immobile, quella metafora di eternità che è retrospezione di Cacciaguida, Firenze che «si stava in pace» (Par. XV, 99): quel verbo mediale ${ }^{25}$, che si richiude in se stesso come un nido, a protezione della propria imperturbabilità. Il tempo che succede è quello dell'entropia, della consumazione, della dissimilitudine dell'oggi (tutte reali sinonimie della decadenza, nella Commedia), di ciò che non resta e non persiste, del male del mutamento, e il termine fatale per Marco Lombardo, «prima che Federigo avesse briga» (Purg. XVI, 117), coincide con quello fino al quale, per dirla invece con Cacciaguida, «'l giglio / non era ad asta mai posto a ritroso / né per divisïon fatto vermiglio» (Par. XVI, 152-154): l'era pre-partitica, quella dell'imperium (Federico II, il giglio bianco: è forza convenire che trattasi di due emblemi/barriere ghibelline...) e pace della civitas, la quale ultima è oggi irraggiatrice di corruzione e discordia, città del demonio.

19 Al centro della rievocazione della Firenze dal «bello/ viver di cittadini» (Par. XV, 130-131) si accampano, come non si è mai sottolineato abbastanza (mi pare), la figura femminile e la cicatrice del cambiamento che, al presente, per Dante la sfregia. L'attacco anaforico dell'analessi cacciaguidiana («Non avea catenella, non corona / non gonne contigiate, non cintura / che fosse a vedere più che la persona», 100-102) si appunta sullo scambio fra merce e persona: poi il «tempo», che fugge la «misura» (104-105: le spose adolescenti, che costringono le famiglie a una corsa, precisamente contro il tempo, per accumular la dote), introduce sùbito il tema dell'ansia dei giorni, del dilapidare i giorni verso guadagni nel vuoto, poiché le ricche «case» sono «di famiglia vòte» (106) e il loro cuore è la «camera» dove «Sardanapalo» (107-108) insegna solo il piacere inane, autoreferente. «Vidi», ripete Cacciaguida (112 e 115): egli così fissa una durata-istante immemoriale, destini fermi di donna, felicemente sottratti a ogni 
tempesta, in comunicazione e in esecuzione di una volontà di provvidenza, la moglie di Bellincion Berti «venir da lo specchio [...] sanza 'l viso dipinto» (113-114), le donne dei Nerli e dei Vecchietti «al fuso e al pennecchio» (117). Una stabile certezza equaziona senza trauma vita e morte, alimenta l'uguaglianza dell'eterno, mentre nell'oggi (brusco, da shock, è qui il ritorno narrativo: "Oh fortunate! Ciascuna era certa/ de la sua sepultura, e ancor nulla / era per Francia nel letto diserta», 118-120) il distacco e la dispersione familiare aprono voragini di oblio, di nostalgia, di diserzione, in cui venga inghiottita un'antica dolcezza (121-126):

L'una vegghiava a studio de la culla,

e, consolando, usava l'idioma

che prima i padri e le madri trastulla;

l'altra, traendo a la rocca la chioma,

favoleggiava con la sua famiglia

d'i Troiani, di Fiesole e di Roma.

Di generazione in generazione, verso la notte dei tempi, favoleggiando... La felicità dell'identico, oggidì rotta e sconciata dalla dissimiglianza, dall'avidità che inventa la carenza. La corsa verso il futuro brucia il giardino che coltivava la donna al centro della casa: è la situazione che marca le indovine nel canto ventesimo dell'Inferno («Vedi le triste che lasciaron l'ago / la spuola e 'l fuso, e fecersi 'ndivine / fecer malie con erbe e con imago», Inf. XX, 121-123). Ancora il «fuso», le opere domestiche, le opere della durata domata, placata, condotta a identità, contrapposte allo sporgersi cupido, irrequieto, verso un futuro che è solum Dei, quindi inconoscibile prima del merito. Asdente, altro divinatore millantatore, "ch'avere inteso al cuoio e allo spago / ora vorrebbe, ma tardi si pente» (119-120), è da Dante colpito da ugual condanna, ma nell'ambito maschile dei mestieri: chi va via, chi prende il largo per cercar di anticipare, di divorare il tempo, perde il posto sicuro, assegnato nella civitas, perde l'addentellato con la concordia laboriosa della comunità.

21 La storia di Manto, in quello stesso canto infernale, ci parla di spazi sterminati, che si apparentano e insieme si oppongono angosciosamente alla vastità del tempo futuro che gli indovini avevano voluto prenotare e disinnescare, come con finezza alludeva Mario Apollonio $^{26}$. La famosa descrizione dell'Italia di «suso» (Inf. XX, 61), che scivola sulle sue acque, quella bellezza ferace, benedetta idealmente da Dio attraverso la sua Chiesa (alla terzina 67-69, il «segnar» dei tre vescovi al centro del lago di Garda), sfocia alfine in un pantano, desertico e mefitico, nel quale le ossa di una maliarda dannata (Manto) fanno da pietra di fondazione di una città. L'erranza di Manto, la fuga dalla città maledetta della Grecia del mito, da Tebe, si conclude in una pozzanghera: tempo perduto e che perde... I luoghi desertici e sterili sono biblicamente associati al maleficio e al demoniaco ${ }^{27}$ : se nell'Antico Testamento i riferimenti sono numerosi per i luoghi «arida» e «inaquosa» e Azazel e il capro sono epifanie del deserto, nel Nuovo Testamento basti pensare alla tentazione di Gesù proprio nel deserto e all'episodio del Geraseno indemoniato. Ma fatalmente, per connotazione, l'infecondità della millantatrice di predizioni si coniuga con quella della donna, cui qui è negata maternità di contro all'attestazione virgiliana nell'Eneide ${ }^{28}$.

Mantova cresce nell'intima negazione del consorzio civile: infeconda vergine è Manto, profuga da una città disintegratasi nell'odio, fuggitivi dalla comunità sono parimenti i suoi servi (lo Scartazzini pensò non per nulla, con oltranza, a spiriti mali, non a uomini) ${ }^{29}$, sbandati sono gli «uomini sparti» (al verso 88) che accorsero sul luogo. E la Mantova del tempo di Dante è una città spopolata, per l'esattezza dalla follia del 
Casalodi che, sobillato da Pinamonte de' Bonacolsi, esiliò i maiores finendo scacciato egli medesimo perché privatosi così di appoggio, come tutti i commenti fin dall'antico informano. «Innuba» $»^{30}$ era definita d'ingresso Manto nella Tebaide, al quarto libro, nella grande scena di evocazione dei morti, che lei officia per il cieco padre Tiresia, e la sua refrattarietà sessuale è segnalata nel settimo libro, dove, nella mischia, a esser saettato da Apollo è fra i tanti, Lampo, che si diceva aver ambito invano una volta a congiungersi con lei (vv. 758-759). Del resto la connotazione sessualmente disforica di Manto pronuncia una femminilità grezzamente biologica, alla Cianghella: Manto in Dante è una sorta di donna dei boschi, dalla nudità oscena e accusata nella pelosità, nudità compagna di quella di Taide (che secondo alcuni commentatori, a mio avviso esatti, è anche volgarmente defecante nel canto diciottesimo infernale) e di quella delle donne di Barbagia e delle «saracine» (103) entro l'invettiva di Forese che, nel canto XXIII del Purgatorio, è rivolta contro le «sfacciate donne fiorentine» che vanno «mostrando con le poppe il petto» (101-102).

Secondo l'assiologia finora da noi delineata, la semplice presenza di Cunizza, non dico in Paradiso ma nel poema, potrebbe risultare seduttivamente enigmatica. Alla sorella di Ezzelino ho dedicato una monografia ${ }^{31}$ ma non ho risolto questo problema, anzi credo proprio di non averlo affrontato. È corrente il rimpianto, da parte della critica, per la grande occasione perduta da Dante: il poeta aveva tra le mani una Maddalena dei tempi suoi, con forti tratti di Francesca epperò sopravvissuta alle draghinasse attraverso avventure degne di un feuilleton ottocentesco con fiammeggiante ambientazione medievale, e di quella figlia dell'amore ha fatto un'algida, sarcastica profetessa di sventura (contro i signori della sua Marca Trevigiana)! Delle peripezie romanzesche di sua vita, due soli versi, che se ne sbarazzano distogliendo alteramente lo sguardo: «ma lietamente a me stessa indulgo / la cagion di mia sorte, e non mi noia» (Par. IX, 34-35). La caotica profondità avventurosa del tempo terreno, ciarpame dell'eterno, è rifiutata $\mathrm{e}$ spazzata via, quasi scostata con gesto infastidito. L'eros che «vinse» (33) Cunizza in vita («il lume d'esta stella», Venere) è sottratto alla contingenza, dunque alla coazione del piacere, della sterile ripetizione, e mutato in amore di «Dio giudicante» (62): il possesso del futuro, da parte di Cunizza, non è illusorio e fraudolento come da parte degli indovini, ma fermo e abolente ogni ansia dell'incognito, ottenuto «leggendo del magno volume / du' non si muta mai bianco né bruno» (Par. XV, 50-51), per dirla con un Cacciaguida. Il godimento della certezza di giustizia e della vendetta divina mostrano, nell'escalation di morte della profezia, convulsa e compiaciutamente oscura, di Cunizza, che copre sei terzine, quasi un'ombra della brama terrena: si appunta il cono d'ombra della Terra, nel cielo dove Cunizza appare a Dante, secondo la teoria di Alfragano ${ }^{32} \mathrm{e}$ ciò, come già osservava Vossler ${ }^{33}$, sembra riflettersi nelle parole delle anime di Venere, così chiaroscurali, così intrise di luce celeste per loro che sono sante e di rezzo terrestre per chi giù resta.

E siamo giunti alla donna che per Dante ogni altra sussume, la Vergine Maria: negli exempla che premettono le espiazioni su ogni balza del monte purgatoriale, essa figura il paradigma della femminilità che, all'inizio di questo saggio ho definito fusionale, o dell'io. In definitiva, è il paradigma del parto: rinuncia, astinenza, defalcamento dell'io, la donna si fa grembo dell'altro, sostrato puro di altra vita. Era il senso irradiante, nel racconto di Cacciaguida, dalla matrona vegliante la culla e affabulante le ragioni e le origini solidali della comunità (le storie «d'i Troiani, di Fiesole e di Roma» [Par. XV, 126]). La visione ideale della donna nella Commedia, composto il quadro di tutte le sue occorrenze attanziali in bono et in malo, fa capo a un sacrificio maieutico, di morte a se 
stessa perché viva la vita. In ogni formella sacra sui gradi del Monte, Maria rinnova gesto $o$ atto di lenizione (in fondo, di smontaggio della situazione, di sdrammatizzazione d'essa per insediarvi corale gloria) affinché si dia evento.

Maria è il trasparente amalgama del divino, indice etico per la femminilità nella Storia. Il suo soffiar vinum non habent è umile preghiera che rappresenta e non esige, nella cornice degli invidiosi, e del peccato che lì si espia ha la modalità inversa dell'obliquità premurosa, discreta a causa del palpitante, dolce patema che tal preghiera possa non trovar ascolto (Purg. XIII, 28). Nella cornice degli iracondi l'accettazione amorevole e, ancora una volta, puramente rappresentativa e non reattiva, è la risposta della mansuetudine, arresa e inclusiva (Purg. XV, 89-91: «"Figliuol mio, / perché hai tu così verso noi fatto? / Ecco, dolenti, lo tuo padre e io / ti cercavamo"»). Agli accidiosi è opposta la sollecitudine di Maria verso Ebron, per visitare Elisabetta (Purg. XVIII, 100): "Maria corse in fretta alla montagna», consumarsi ineroico nel fare. Agli avari si ricorda (Purg. XX, 19-24) la povertà dell'«ospizio» al parto di Maria (della «dolce» Maria: l'epiteto si accompagna quasi sistematicamente al nome o ai fatti della Vergine): ancora una volta la spoliazione di sé che permette l'Altro. Ai golosi (Purg. XXII, 142-144) ancora le nozze di Cana sono esibite a monito, stavolta quali esempio di frugalità, che, ancora, è un annullarsi perché l'essere d'altro si dia (le «nozze orrevoli e intere» [143], dove l'attenzione è pertanto posta da Dante, e dev'esser posta dal lettore, al secondo aggettivo). Infine, la castità chiude il cerchio, poiché la scena allusa dell'annunciazione (Purg. XXV, 128) rimanda al primo exemplum della serie (Purg. X, 34-45), in cui solo l'angelo compariva, sicché adesso lo schermirsi della Vergine, virum non cognosco (ripetuto in coro da «spirti per la fiamma andando» [Purg. XXV, 124]), associa pudore a umiltà, virtù negletta appunto dai penitenti della prima balza del Monte: un grande moto circolare di astensione, un ritrarsi che crea, ordina il ciclo purgatoriale.

Il negarsi accogliente (potente ossimoro dell'Incarnazione) di Maria, che valse ai figli di Adamo il perdono del peccato originale, fa insomma luce al cammino verso ogni perdono successivo, individuale. La parola di donna affonda dunque, nel mentre che emerge, in un silenzio demiurgico: lo capiamo anche soltanto da quel modello supremo di genere per Dante. Dalle citazioni evangeliche alla visione diretta: dopo la preghiera di San Bernardo, nell'ultimo canto paradisiaco, l'intercessione della Madonna è un puro volger d'occhi. La gloria di Maria è tutta un tramite, è tutta un far riconoscere le ragioni dell'unità uscendo d'essa per realizzarla. La divisoria straripanza verbale di Francesca, all'inizio del libro, è in clamorosa contrapposizione a questo (Par. XXXIII, 40-45):

Li occhi da Dio diletti e venerati,

fissi ne l'orator, ne dimostraro

quanto i devoti prieghi le son grati;

indi a l'etterno lume s'addrizzaro,

nel qual non si dee creder che s'invii

per creatura l'occhio tanto chiaro.

Beatrice, emissaria di Maria, e Matelda, di Beatrice prefigurazione e antesignana, nell'Eden accusano invece Dante della distanza temporale da lui inferta come un taglio alla propria vita, dell'abbandono che il viator ha fatto di se stesso, della sua virtualità. Esse, che hanno raggiunto l'identità, a sé uguale, dell'eterno, mettono a nudo il peccato che nella Commedia abbiamo visto affliggere spesso la femminilità: l'insolvenza, il cedimento al tempo secolare, la diaspora nel tempo, il rimpianto dal volto di Medusa... Dopo che la distanza simbolica frapposta dal fiume d'oblio Lete ha prodotto uno struggente riflusso dal passato col fantasma di giovinezza Matelda ${ }^{34}$, la commozione 
cede al giudizio, cede all'interrogazione su quell'oblio di sé che ora è dissipato ma che chiede nuovo oblio (il battesimo per mano di Matelda dentro il Lete) perché sia rimosso come colpa: a riprova, come vuole Paul Ricœur, che l'oblio è impigliato nella memoria e che ne è anzi la condizione ${ }^{35}$, fuor di patologia per il moderno, guariti nell'anima per Dante. È allora che si dà una sorta di scambio tra quel che è il femminile archetipale per Dante e il maschile del viator, è allora che Beatrice compare «proterva» (Purg. XXX, 70), comparata a un "ammiraglio» (58) che impartisca concitati ordini alla ciurma, mentre Virgilio, svanito, diventa «mamma» (44) al sentire di Dante e un pianto femmineo accompagna sia l'angoscia per le accuse di Beatrice al passato del viator sia la resipiscenza di questi, la cui virilità è derisa sotto specie della «barba» (Purg. XXXI, 68). Il tempo, che scorre castigatore e la cui sferza è il rimorso, segnando gap all'interno della persona (una declinazione morale, in fondo, della concezione agostiniana, la cui «mnemoteologia» ${ }^{36}$ vuole il tempo esistere soltanto nell'interiorità, esser dato coscienziale), ha fatto della vita di Dante una selva oscura, come da incipit del poema, ovvero, tal quale afferma in Eden Beatrice, un «terren» che è «maligno» e "silvestro» (Purg. XXX, 118-119), proprio come la sterile piana di Manto.

Spazio metafora del tempo vano, inutilizzato o luogo di «via non vera» (Par. XXX, 130): ma stavolta è la donna a rimproverarlo all'uomo, la Gentilissima a Dante. Se vale l'ironica definizione di bovarismo che Edoardo Sanguineti ${ }^{37}$, radicalizzando la lettura del personaggio da parte di Contini, assegnò a Francesca da Rimini, Dante può ben dire, quindi, "Francesca c'est moi !». E in questa androginia, che è universalità di visione dell'umano, Dante riscatta (se la cancel culture non prevarrà...) certi automatismi misogini, storicamente funzionali alla sua reazione morale e al suo disegno politico, ma dietro i quali (credo o almeno spero di averlo dimostrato) vi è un amore che cerca forma più vera.

\section{NOTE}

1. G. W. F. Hegel, Estetica [Aesthetik], a cura di N. Merker, Torino, Einaudi, 1976 ${ }^{4}, 2$ voll., vol. II, pp. 1235-1236.

2. Cfr. G. Contini, Lettre d'Italie (1945) [1971], in Id., Altri esercizi (1942-1971), Torino, Einaudi, 1972, pp. 267-293: p. 290.

3. Per la personificazione femminile della polis in Dante, $\mathrm{cfr}$. le poche pagine, ma calibratissime e generose di rassegna bibliografica, in E. Brilli, Firenze e il profeta. Dante fra teologia e politica, Roma, Carocci, 2012, pp. 223-229.

4. Purg. X, 95. Ogni citazione dalla Commedia dantesca si intende riferita a La Commedia secondo l'antica vulgata, a cura di G. Petrocchi, Firenze, Le Lettere, 1994² [1966-1967], 4 voll. Le citazioni dai commenti alla Commedia, a partire da quelli secolari fino a quello di Hollander, faranno invece capo, senza altro onere di rimando bibliografico, al benemerito sito del Dartmouth College, $<$ https://dante.dartmouth.edu>. Per le citazioni bibliche, ogni rinvio è a Biblia Sacra Vulgatae Editionis Sixti V Pontificis maximi iussu recognita et Clementis VIII auctoritate edita, Romae, Editiones Paulinae, 1957. Le sigle adottate per i singoli libri sono quelle definite, in appendice al Catechismus Catholiace Ecclesiae, sul sito vaticano <www.vatican.va/archive/catechism_lt/abbrev_lt.htm>. 
5. Cfr. G. Petrocchi, Dante e San Bernardo, in Id., L'ultima dea, Roma, Bonacci, 1977, pp. 137-155, a p. 145. Generico il vecchio rimando crociano a Giotto (cfr. B. Croce, L'ultimo canto della "Commedia», in Id., Poesia antica e moderna. Interpretazioni, Bari, $1943^{2}$, pp. 151-161, a p. 159), da cui sembra prender le orme G. Fallani, Dante e la cultura figurativa medievale, Bergamo, Minerva Italica, $1976^{2}$, pp. 160-161, con rimando altrettanto sfuocato mentre, nella lettura diretta del canto ultimo paradisiaco, da parte del medesimo (Id., Il canto XXXIII, in Paradiso. Letture degli anni 1979-'81, Roma, Bonacci, 1989, pp. 851-852) l'affastellamento casuale dei modelli ecfrastici va dalle catacombe di Priscilla ad Arnolfo, con scarso utile. L. Pasquini, «Pigliare occhi, per aver la mente». Dante, la Commedia e le arti figurative, Roma, Carocci, 2020, pp. 200-208, offre un ampio ventaglio di proposte bizantine ravennati, anche suggestive, ma senza reale scelta orientativa.

6. Su ciò, si vedano le recentissime e illuminanti pagine del capitolo T. Klinkert, La memoria del dolore nella Commedia, in Id., La modernità di Dante. Prospettive semiotiche sulla Commedia, Ravenna, Longo, 2021, pp. 53-77.

7. Vedi, a cura della sua scuola, le Vertical Readings of Dante's Comedy, a cura di G. Corbett e

H. Webb, Cambridge, Open Book Publishers, 2016, 3 voll.

8. Basterà al proposito, senza perdersi da sede qui impropria nella giungla ormai fittissima della bibliografia critica ildegardiana, M.-M. Davy, Il simbolismo medievale [Initiation à la symbolique romane (XII siècle), 1964], Roma, Edizioni Mediterranee, 1988, pp. 175-177.

9. L. Charbonneau-Lessay, Le bestiaire du Christ, Paris, Albin Michel, 2006 ${ }^{3}$, p. 185.

10. Cfr. Sant'Agostino, Commento ai Salmi, a cura di M. Simonetti, Milano, Fondazione Lorenzo Valla / Arnoldo Mondadori, 2011, p. 537 (In psalmum CXLIII enarratio, sermo ad plebem).

11. Per le corrispondenze quinarie e per la simbologia di esse dentro il poema dantesco, cfr. L. De Poli, La structure mnémonique de la Divine Comédie. L'ars memorativa et le nombre cinq dans la composition du poème de Dante, Bern, Peter Lang, 1996, soprattutto pp. 129-157.

12. Per questa di Pia, come per le altre 'lapidi' della Commedia, il rimando ormai canonico è a S. Carrai, Quando ti gioverà dicere «I' fui». Lo stile epigrafico nella «Commedia», in Id., Dante e l'antico. L'emulazione dei classici nella «Commedia», Firenze, Edizioni del Galluzzo per la Fondazione Ezio Franceschini, 2012, pp. 43-86.

13. Nel suo libro di alta divulgazione uscito postumo, Marco Santagata collega il peccato d'invidia a quello della balza precedente dove, tra i superbi, espia il nipote di Sapìa, oggetto del suo invido risentimento, Provenzano Salvani. I due peccati si rovesciano l'uno nell'altro, come dice Santagata, per radice comune di perversione della legge di amore e di solidarietà in terra (cfr. M. Santagata, Le donne di Dante, Bologna, Il Mulino, 2021, pp. 174-175). I due senesi, zia e nipote, in certo modo si purgano di rinfaccio, specchiandosi a vicenda, da un girone all'altro, le loro passate vergogne e affinandosi sopra di esse.

14. H. Weinrich, Lete. Arte e critica dell'oblio [Lethe. Kunst und Kritik des Vergessens, 1997], Bologna, Il Mulino, 1999, p. 39.

15. «L'elogio [...] della "vedovella" pudica e fedele post mortem sarà una palinodia remuneratrice delle laide rivelazioni presenti» (cfr. Dante Alighieri, Rime, a cura di G. Contini, Torino, Einaudi, $1980^{4}$, p. 83, nota 2).

16. Che citiamo da Dante Alighieri, Vita nuova. Rime, a cura di M. Grimaldi e D. Pirovano, Roma, Salerno, 2015-2019, 2 voll., vol. II, pp. 815-819.

17. Su questo filone resta fondamentale J. B. Percan, Femina dulce malum. La donna nella letteratura medievale latina (secoli X-XIV), Roma, Kappa, 2003.

18. Conosciuta da Dante quand'egli fu ospite, nei primi tempi del suo esilio, del marito Guido Salvatico, che era legato alla parte nera fiorentina, onde Santagata ipotizza regolamento di conti, da parte di Dante (cfr. Santagata, Ledonne di Dante, cit., p.188), per l'esito nullo dell'abboccamento del poeta coi guelfi neri: uno dei tanti esempi di lettura tattica e contingentista del poema, secondo il tipo introdotto da U.Carpi, La nobiltà di Dante, Firenze, Polistampa, 2004, 2 voll., che Santagata spesso insegue da presso. Più convincente (Santagata, 
Le donne di Dante, cit., p. 184) è il compianto studioso nella sottile annotazione che alla solitudine della morte di Bonconte fa replica la sua solitudine nel gruppo antipurgatoriale dei morti di morte violenta («io vo tra costor con bassa fronte», Purg. V, 90), perché abbandonato nelle preghiere (quasi, direi, un carcerato che non riceva mai visite...), e che in terra all'oblio del corpo di Bonconte risponde l'oblio della sua anima.

19. G. Padoan, Nelle cornici degli accidiosi e degli avari, in Lectura Dantis Modenese, Modena, Banca Popolare dell'Emilia, 1985, 3 voll., vol. II, pp. 83-99, a p. 93.

20. Su Dante lucchese è tutt'oggi la miglior bussola, per gli studiosi, G. Varanini, Dante e Lucca, in Dante e le città dell'esilio, Atti del Convegno internazionale di Studi, Ravenna (11-13 settembre 1987), direzione scientifica di G. Di Pino, Ravenna, Longo, 1989, pp.91-114. Ma importanti aggiornamenti, seppur di sintesi e di rassegna, in A. Barbero, Dante, Bari, Laterza, 2020, pp. 223-224, e in P. Pellegrini, Dante Alighieri. Una vita, Torino, Einaudi, 2021, pp.125-126. La proposta di G. Inglese, Una biografia impossibile, in Forum. Dante and Biography, a cura di E. Brilli, «Dante Studies», 136, 2018, p. 165, che l'allusione a Gentucca, da parte di Dante, palinodii la sardonica invettiva antilucchese del XXI dell'Inferno (al modo in cui Nella del Purgatorio riscatta la tenzone giovanile con Forese) attestando una seconda sosta di Dante esule in Lucca, negli anni di Castruccio Castracani trionfante, avrebbe se non altro il fascino di implicare la mise en abîme di un purgatorio nel purgatorio per l'auctor.

21. V. L. Puccetti, Quale Stazio per Dante?, in P. Allegretti e M. Ciccuto (a cura di), I classici di Dante, Firenze, Le Lettere, 2017, pp. 215-239, a p. 238.

22. Cfr. Dante, Vita nuova. Rime, cit., vol. I, p. 157 (Vita nuova XIX, 1).

23. Puccetti, Quale Stazio per Dante?, cit., pp. 238-239.

24. Ad locum.

25. Se accettiamo l'interessante proposta, estensiva della diatesi media alla marca pronominale non riflessiva, di E. Jezek, Classi di verbi tra semantica e sintassi, Pisa, ETS, pp. 130 sgg. Nel nostro caso, la classificazione tecnica di riflessivo inerente, quale mera, inargomentale conferma della predicazione del verbo (cfr. Grammatica dell'italiano antico, a cura di G. Salvi e L. Renzi, Bologna, Il Mulino, 2010, 2 voll, vol. I, pp. 450 sgg.), lascia insoddisfatti. La sorpresa di Rohlfs, dinanzi alla presenza della marca pronominale coi verbi intransitivi in italiano (G. Rohlfs, Grammatica storica della lingua italiana e dei suoi dialetti [Historische Grammatik der Italienischen Sprache und ihrer Mundarten, 1949], Torino, Einaudi, 1968, 3 voll., vol. II, p. 188), di cui pur Dag Norberg aveva ricostituito la filiera storico-evolutiva nel basso latino (cfr. D. Norberg, Syntaktische Forschungen auf dem Gebiete des Spätlateins und des frühen Mittellateins, Uppsala, Lundequistska Bokhandeln und Leipzig, Harassowitz, 1943, pp. 152-174), non è meno indiziaria del freddo rilievo, compiuto da altri, che in italiano antico essere e stare si fanno riflessivi soprattutto in poesia (Grammatica dell'italiano antico, cit., p. 459). Occorre sempre diffidare di interpretazioni preventivamente e affettivamente stilistiche di fatti di lingua, ma (chiediamo) c'è altro modo di intendere il verso dantesco che il ripercuotersi di uno stare come evento sopra il soggetto di quello stare medesimo, oltre l'intenzione dell'agente? Dante non ci dice che, stando, si finisce con lo stare? Che la quiete si autogenera?

26. Cfr. M. Apollonio, Dante. Storia della Commedia, Milano, Vallardi, 1951, 2 voll., vol. I, p. 629.

27. Cfr. I. Baldelli, Il segno del demonio sui maghi e sugli indovini [pp. 1-15], in Id., Studi danteschi, a cura di L. Serianni e U. Vignuzzi, Spoleto, Centro di Studi sull'Alto Medioevo, 2015, pp. 13-14.

28. Virgilio recisamente smentisce ogni altra versione delle origini della sua città («la verità nulla menzogna frodi», al verso 99) e cioè, come vien detto fin dai primi commentatori, smentisce se stesso, che nel decimo libro dell'Eneide (vv. 198-203), nella rassegna delle navi del contingente italico che partono con Enea a dar man forte contro Turno, cita Ocno, figlio di Manto e del Tevere, cui attribuisce la fondazione di Mantova, col nome della madre (si cita da: P. Vergili Maronis, Opera, post R. Sabbadini et A. Castiglioni recensuit M. Geymonat, Torino, Paravia, 1973). Dal D'Ovidio fino ad Hollander si è sostenuto che l'accanimento di Virgilio contro gli indovini e la 
presa di distanze da se stesso, dal sé irretito nel paganesimo, avesse lo scopo di coprire o cancellare la fama medievale di Virgilio mago-profeta, documentata dal classico studio ottocentesco del Comparetti e innescata dalla misinterpretazione della quarta egloga, quella del puer divino.

29. Al verso 463: si cita da P. Papini Stati, Thebaidos libri 12, recensuit et cum apparatu critico et exegetico instruxit D. E. Hill, Leiden, Brill, $1996^{2}$.

30. Vedi il commento ad locum.

31. V. L. Puccetti, Fuga in Paradiso. Storia intertestuale di Cunizza da Romano, Ravenna, Longo, 2010.

32. Allusa ai vv. 117-118 del canto, dall'anima beata di Folchetto di Marsiglia: «Da questo cielo in cui l'ombra s'appunta / che 'l vostro mondo face».

33. Cfr. K. Vossler, La Divina Commedia studiata nella sua genesi e interpretata [Die göttliche Komödie, 1910], Bari, Laterza, 1983², 4 voll., vol. IV, pp. 172-173.

34. Su ciò mi permetto di rinviare al mio recente V. L. Puccetti, Gli inverni fiorentini e la primavera di Matelda, Ravenna, Longo, 2020.

35. P. Ricœur, La memoria, la storia, l'oblio [La mémoire, l'histoire, l'oubli, 2000], Milano, Cortina, 2003, p. 607.

36. Secondo l'arguta formula di Weinrich, Lete. Arte e critica dell'oblio, cit., p. 51.

37. Cfr. E. Sanguineti, Il realismo di Dante, Firenze, Sansoni, 1966, p. 64.

\section{RIASSUNTI}

Nel saggio l'autore indaga la dimensione temporale nella costruzione dei personaggi femminili della Commedia dantesca e la interpreta come marca di un'inadempienza morale, come tempo dell'abbandono e del rimpianto, per la donna, di distanza frammessa col compito che Dante ideologicamente assegna alla figura femminile dentro la polis, quello di promuovere, senza accampare la propria individualità, la continuità e l'amalgama dei valori comunitari, di garantire la stabilità e la concordia sociali con la fedeltà, con un tempo senza intervalli che prefigura l'eterno. Il modello di questa demiurgia schiva al femminile è costituito dalla Santa Vergine, come da episodi evangelici esemplificatori posti all'ingresso di ogni cornice del purgatorio. Nel finale del saggio si mostra come Dante, nel paradiso terrestre, su di sé assuma ed espii, ricevendo i rimproveri conformi di Beatrice e immergendosi nel Lete dell'oblio, il peccato che imputa alle donne dannate o espianti del poema, quello della rottura del tempo unitario, dello spreco che è nel distacco e nella solitudine.

Dans cet essai, l'auteur interroge la dimension temporelle dans la construction des personnages féminins de la Divine Comédie de Dante et l'interprète comme la marque d'un défaut moral, comme un temps d'abandon et de regret pour la femme, une distance interposée à la tâche que Dante attribue idéologiquement à la figure féminine, au sein de la polis, celle de favoriser la continuité et l'amalgame des valeurs communautaires, sans faire prévaloir sa propre individualité, et de garantir la stabilité et l'harmonie sociale avec fidélité, avec un temps sans intervalles, qui préfigure l'éternel. Le modèle de cette discrète démiurgie féminine est celui de la Vierge Marie, comme peuvent l'illustrer les épisodes évangéliques placés à l'entrée de chaque corniche du Purgatoire. À la fin de l'essai, on montre comment Dante, dans le paradis terrestre, assume et expie lui-même, recevant les reproches conformes de Béatrice et se plongeant dans le 
Léthé de l'oubli, le péché qu'il attribue aux femmes damnées ou expiantes dans le poème, celui de la rupture du temps unitaire, du gaspillage qui est dans le détachement et la solitude.

In this essay, the author investigates the temporal dimension in the construction of the female characters of Dante's Comedy and interprets it as a mark of a moral default, as a time of abandonment and regret, for the woman, of distance intertwined with the task that Dante ideologically assigns to the female figure within the polis, that is promoting the continuity and amalgamation of community values, without encumbering her own individuality, and guaranteeing stability and social harmony with fidelity, with a time without intervals, that prefigures the eternity. The model of this shy feminine demiurgy is constituted by the Holy Virgin, as in exemplifying evangelical episodes placed at the entrance of each frame of Purgatory. In the end of the essay it is shown how Dante, in the Earthly Paradise, assumes and expiates upon himself, receiving the compliant reproaches of Beatrice and immersing himself in the Lethe of oblivion, the sin that he attributes to the damned or expiant women of the poem, that of the breaking of the unitary time and of the waste that is in detachment and solitude.

\section{INDICE}

Keywords : time, memory, Virgin Mary, medieval misogyny, suffrage prayers

Parole chiave : tempo, memoria, Vergine Maria, misoginia medievale, preghiere di suffragio

Mots-clés : temps, mémoire, Vierge Marie, misogynie médiévale, prières de suffrage

\section{AUTORE}

\section{VALTER LEONARDO PUCCETTI}

Valter Leonardo Puccetti è docente di Letteratura italiana presso il Dipartimento di Studi Umanistici dell'Università del Salento. Specialista di Dante e in genere di letteratura italiana medievale, estende le sue ricerche alla letteratura italiana dell'otto-novecento e a temi di teoria e di comparatistica letterarie. È recente il suo volume, per l'editore Longo, Gli inverni fiorentini e la primavera di Matelda (Ravenna, Longo, 2020).

Valter Leonardo Puccetti enseigne la littérature italienne au Département d'études humanistes de l'Université du Salento. Spécialiste de Dante et de littérature italienne du Moyen Âge, il a élargi son domaine de recherche à la littérature italienne des $\mathrm{XIX}^{\mathrm{e}}$ et $\mathrm{Xx}^{\mathrm{e}}$ siècles, ainsi qu'à la théorie littéraire et à la littérature comparée. Parmi ses dernières publications, Gli inverni fiorentini e la primavera di Matelda (Ravenne, Longo, 2020). valter.puccetti@unisalento.it 